\title{
Positive Effects of Diphlorethohydroxycarmalol (DPHC) on the Stability of the Integument Structure in Diet-Induced Obese Female Mice
}

\author{
Chae-lim Kim ${ }^{1}$, Sun-yeong Cha ${ }^{1}$, Min Young Chun ${ }^{2}$, Bumsoo Kim ${ }^{3}$, Min Young Choi ${ }^{4}$ \\ and ${ }^{\dagger}$ Yong-Pil Cheon ${ }^{1}$ \\ ${ }^{I}$ Division of Developmental Biology and Physiology, School of Biosciences and Chemistry, \\ Institute for Basic Sciences, Sungshin Women's University, Seoul 142-742, Korea \\ ${ }^{2}$ Global Medical Science, Sungshin Women's University, Seoul 142-742, Korea \\ ${ }^{3}$ Exercise Rehabilitation and Welfare, Sungshin Women's University, Seoul 142-742, Korea \\ ${ }^{4}$ Industrial Design, Sungshin Women's University, Seoul 142-742, Korea
}

\begin{abstract}
Diphlorethohydroxycarmalol (DPHC) is a known to modulate the expression of extracellular matrix (ECM) components in 3T3-L1. However, the possible role of DPHC in integument stability during obesity induction is not clear yet. We evaluated the effects of DPHC on collagen or elastic fiber quantity in integument during obesity induction with high-fat diet. The dorsal back integument sections were stained with hematoxylin-eosin, Masson trichrome, and Verhoff-Van Gieson. The intensities of collagen fibers and elastin fibers were analyzed with ImageJ. The number of fibroblasts was counted at $\times 1,000$ fields. The number of fibroblast was increased by obesity induction, but DPHC suppressed it in a concentrationdependent manner both in lean and obese mice. On the other hand, the intensities of collagen fibers were increased by DPHC treatment in obese mice groups but not in lean mice groups. The intensities of collagen fibers of obese mice were lower than that of the lean mice in $0 \%$ group. However, the number became similar between lean and obese mice by the treatment of DPHC. The intensity of elastic fibers was increased in the lean mice with the concentration of DPHC. In the obese mice group, there were increasing patterns but only significant at $10 \%$ DPHC group. The intensity of elastic fibers of obese mice was higher than lean mice in $0 \%, 1 \%$, and $10 \%$ groups. Histologically epithelial cells and follicle cells which were diffused nuclear staining forms were increased by DPHC treatment. The results suggest that the activity of integument cells during obesity induction can be modulated by DPHC.
\end{abstract}

Key words : Diphlorethyohydroxycarmalol, Integument, Collagen fibers, Elastic fibers, Obesity induction

\section{INTRODUCTION}

By the anatomical site, the thickness of integument varies, and within one region it will vary depending on physiological parameters such as hair cycle (Azzi et al.,
2005; Mecklenburg et al., 2013). It was also affected by age and various integument diseases (Mecklenurg et al., 2013). Although the structure of mouse integument is different from the human, it is useful to understand the role of various chemicals as regulator in integument. It has

\footnotetext{
Manuscript received 30 June 2015, Received in revised form 9 July 2015, Accepted 23 July 2015

${ }^{\dagger}$ Corresponding Author : Yong-Pil Cheon, Division of Developmental Biology and Physiology, School of Biosciences and Chemistry, Sunghsin Women's University, Mia-Unjeong-Green Campus, 147 Mia-dong, Kangbuk-gu, Seoul 142-742, Republic of Korea. Tel. : +82-2-920-7639, Fax : +82-2920-2093, E-mail : ypcheon@sungshin.ac.kr

This is an Open Access article distributed under the terms of the Creative Commons Attribution Non-Commercial License (http:// creativecommons.org/licenses/by-nc/3.0) which permits unrestricted non-commercial use, distribution, and reproduction in any medium, provided the original work is properly cited.
} 
been evaluated that the stability of integument depends on the ability of basement layer of epidermis and the activities of dermal cells.

Mouse integument can be separated into the epidermis and dermis (containing cutaneous adnexa) and subcutis (penniculum carnosum) (Murillas et al., 1995; Mecklenburg et al., 2013). Such as hair follicles, sebaceous glands, and eccrine glands which are observed in dermis structure are derived from the ectoderm. Primary hair follicles have large sebaceous glands, prominent innervations, and prominent blood supplies. In contrast, secondary hair follicles are smaller and have small sebaceous glands. Secondary hair follicles are the predominant type (Meyer, 2009). Sebaceous glands are derived from the follicular isthmus and are composed of a glandular and a ductal component, but sweat gland is absent in mouse (Taylor et al., 2012). Dermis and subcutis (synonyme: hypodermis, panniculum carnosum) originates from mesoderm and provides the tensile strength to integument, elasticity and flexibility. They are loose connective tissues and have much extracellular matrix (ECM). The hypodermis is characterized by densely-packed adipocytes. This adipose tissue regulates regeneration homeostasis of epidermis, dermis, and cutaneous appendages (Cassisa, 2013). ECM of integument is important to preserve tissue integrity, but is also required to deposit and remodel the ECM itself and to communicate with the cell behavior (Eckes et al., 2014).

The ECM of the dermis mainly consists of type I, III, V, and VI collagen fibers, reticular and elastic fibers, and dermal ground substance (glycosaminoglaycans, proteoglycans, hyaluronic acid, and dermatan sulphate). Collagen and elastin are the structure of the skin that maintains both elasticity and firmness. Fibroblast cells create both collagen and elastin matrix that can be activated by glycolic acid and other peels. The special fibroblast cells are found in the papillary level of the second layer of the skin (dermis). Many creams and lotions are marketed to promote colla- gen formation by fibroblastic activation.

The loss of molecular integrity of the dermis leads to increased rigidity, decreased torsion extensibility (Martini, 2004), and diminished elasticity (McCallion \& Li, 1993). Previously, we showed that DPHC modulate the expression profiles of ECM components such as collagen type 1 and collagen type 2 (Jeon et al., 2013). Diphlorethohydroxycarmalol (DPHC) isolated from Ishige okamurae, is a polyphenolic compound (Stern et al., 1996), and has antiinflammatory and anti-diabetic activity (Heo et al, 2009). The interaction of cell and its surrounding matrix is important in repair and scar of dermis (Eckes et al., 2010) and the stability of the integument depends on the physiological conditions. The purpose of this study was the evaluation of the DPHC effects on the structural stability of integument. The distribution and quantity of extracellular fibrils was evaluated during the increase of integument adipose tissues.

\section{MATERIALS AND METHODS}

\section{Chemicals and reagents}

DPHC derived from I. okamurae was used. Lotion, a cosmetic formulation, was employed to transfer the DPHC into integument (Jeon et al., 2013). A mixture of propylene glycol $(0.01 \%)$ and menthyl lactate $(1.6 \%)$ was used as medium.

\section{Animals and treatment}

All experimental animal studies followed to the Guide for the Care and Use of Laboratory. Animal were maintained under standard conditions at Sungshin Women's University. Four-week-old C57BL/6J female mice were purchased from the Charles River Laboratories and allowed to adapt to the experimental conditions for 10 days. Total 70 animals were randomly divided in seven groups: lean control, obesity control, DPHC $(0 \%, 0.01 \%, 0.1 \%, 1 \%$, 
and $10 \%$; weight; volume). Mice were individually housed in cages with $14 \mathrm{~h} / 10 \mathrm{~h}$ of light/dark cycle (light on at 06:00). Obesity control and DPHCs-treated mice were exposed to a high fat hypercaloric diet (60\%). Lean control mice were allowed free access to standard food (Purina). Food consumption and weight gain were measured daily and weekly, respectively. Body weight and LEE Index were used to the criteria for obesity. Evaluation of obesity levels are more than 300 when designated as obesity (Dubuc, 1976). After 6 weeks of feeding, the hairs on the dorsal of back were fleeced and DPHC containing media were applied to the skin and spread over the area once a day during 28 days.

\section{Histology and collagen and elastin staining}

After being sacrificed, the dorsal integument samples from the treated area were taken and fixed in $4 \%$ buffered paraformaldehyde and routinely embedded in paraffin: put the fixed tissue speci-mens into the automatic infiltration machine (EG1150H, Leica, Germany) and dehydrate it by rising alcohol series. Through the xylene replacement process tissues were embedded with paraplast (Cat \#: P3558 Sigma-Aldrich, USA). After that, serial sections of integument samples were made vertically from central region by using rotary microtome (RM2245, Leica, Germany) at $4 \mu \mathrm{m}$ thickness. Sections were attached onto the glass slide on its exact position and dried in the $42^{\circ} \mathrm{C}$ slide warmer for 6 hour, and then deparaffinized by xylene. And they were stained by hematoxylin-eosin, Masson trichrome for collagen fibers, and Verhoff-Van Gieson for elastic fibers.

The stained tissues were observed under the light microscope (Nikon, Japan). For image analysis the freeware ImageJ (National Institutes of Health software) v1.33 and the Color Histogram plug-in, both downloaded from the $\mathrm{NIH}$ website (http://rsb.info.nih.gov/ij) were used. After getting 13 image per group randomly, we divided them into the total area of the observed video and calculated the destiny of collagen fiber or elastin fiber as a $\mathrm{mm}^{2}$ per unit area. Staining intensity and percentage of field positive was quantitated. Each slide was digitized using an automated imaging system that produced an image of every dot and also informed the dot coordinates on the slide. The number of fibroblast was counted under $\times 1,000$ field and repeated 10 times randomly.

\section{Statistical analysis}

All measurements were performed at least 10 times repeatedly and expressed as means \pm SD. The significance of differences among groups was determined by using 1way ANOVA followed by $t$-test (performed using SPSS software). $P<0.05$ value was considered as statistically significant.

\section{RESULTS}

\section{Topical treatment of DPHC decreased the number} of fibroblast

The amount of the ECM is dependent on the activity and number of fibroblast. In lean mice, the number of fibroblast within given area was not different until 1\% DPHC treatment but was significantly decreased by $10 \%$ DPHC treatment. Such decrease was also observed in obese mice (Fig. 1). The difference between the lean and the obese mice was only observed in $0 \%$ and $0.01 \%$ DPHC treatment groups but not in other concentration groups (Fig. 1).

\section{The intensity of collagen fibers was increased in DPHC-treated group}

In the lean mice group, the intensity of collagen fibers was not different between DPHC treatment groups. After obesity induction, the intensities of collagen fibers increased with the increase of DPHC concentration. Statistical significance was detected from $0.1 \%$ DPHC group (Fig. 2). 


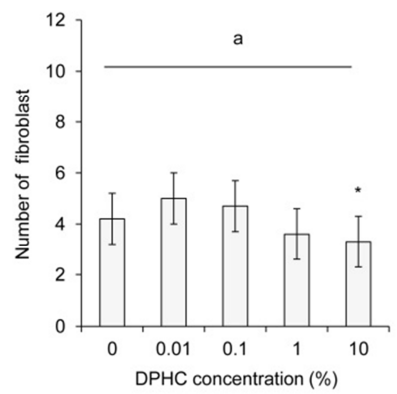

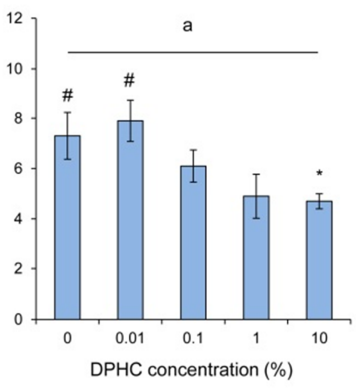

Fig. 1. Changes in the number of fibroblast in dermis before and after of diet-induced obesity. (A) lean and (B) obesity mice. The numbers of fibroblasts were counted for 20 fields of 6 sections at high power field $(\times 1,000)$ and presented as Mean $\pm \mathrm{SD}$, a: $P<0.05$, one-way ANOVA. $* P<0.05,0 \%$ DEPC vs other DEPC group within lean or obesity mice, \# $p<0.05$ lean vs obesity of same dosage of DPHC.

A

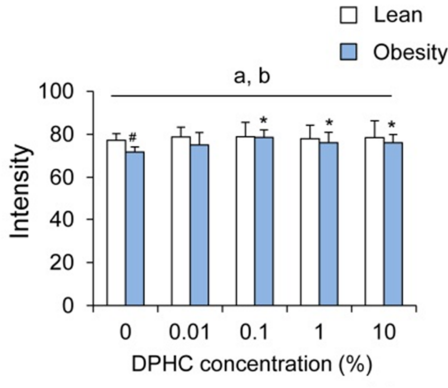

B

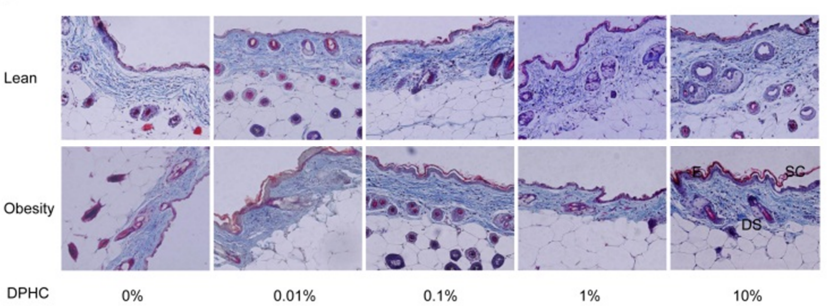

Fig. 2. Changes in intensity of collagen fibers of dorsal back integument in a given area of lean and obesity mice. (A) Intensity of collagen fibers was analyzed using ImageJ v1.33 and the Color Histogram plug-in. (B) Micrograph of the skin section stained with Masson trichrome staining $(\times 100$, (insertion, $\times 400)$ ). Collagen fiber stained blue. Keratin, cytoplasm, and muscle stained red Abbreviation: $\mathrm{SC}$, stratum corneau; E, viable epidermis; DS, dermis and subcutis.

Differences of intensity in same concentration groups of lean and obese mice were observed in 0\% DPHC groups but not in other DPHC treated groups (Fig. 2).

3. The amount of elastin fibers was increased in DPHC-treated group

The intensity of elastic fibers was increased in lean mice with the concentration of DPHC. In the obese mice group, the intensity of elastic fibers had increasing patterns but was only significant at $10 \%$ DPHC group. Intensity of elastic fibers was decreased significantly by $0.01 \%$ DPHC treatment $(P<0.05)$. In $0.1 \%$ DPHC and $1 \%$ DPHC groups, it was similar with 0\% DPHC treatment group (Fig. 3). Intensity of elastic fiber of obese mice was higher than lean mice in $0 \%, 1 \%$, and $10 \%$ groups. However, the

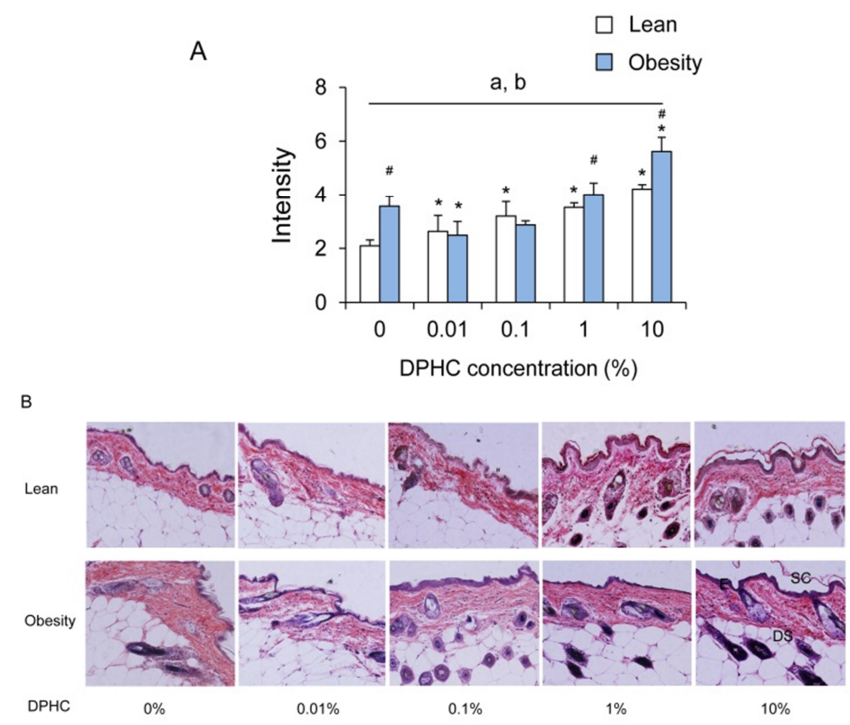

Fig. 3. Changes in intensity of elastin fibers of dorsal back integument in a given area of lean and obesity mice. (A) Intensity of elastin fibers was analyzed using ImageJ v1.33 and the Color Histogram plug-in. (B) Micrograph of the skin section stained with Verhoff-Van Gieson staining $(\times 100$, (insertion, $\times 400))$. Elastic fiber stained blueblack to black. Collagen stained red. Nuclei stained blue to black. Abbreviation: SC, stratum corneau; E, viable epidermis; DS, dermis and subcutis. 
intensity was not different between the lean and the obese mice in $0.01 \%$ and $0.1 \%$ groups.

\section{Modulation of the histological characters of inte-} gument by DPHC-treated group

Epithelium was stained strongly in the DPHC treatment mice both in lean and obese mice. The basal epithelial layer continuously constructed 1 or 2 cell layers and the keratinizing cell formed 1-2 layers. The size of epidermal keratinocytes increased and the nucleus displaced to the periphery of the cell. DPHC increased thickness of noncornified epidermal layers and increased the size of nucleated
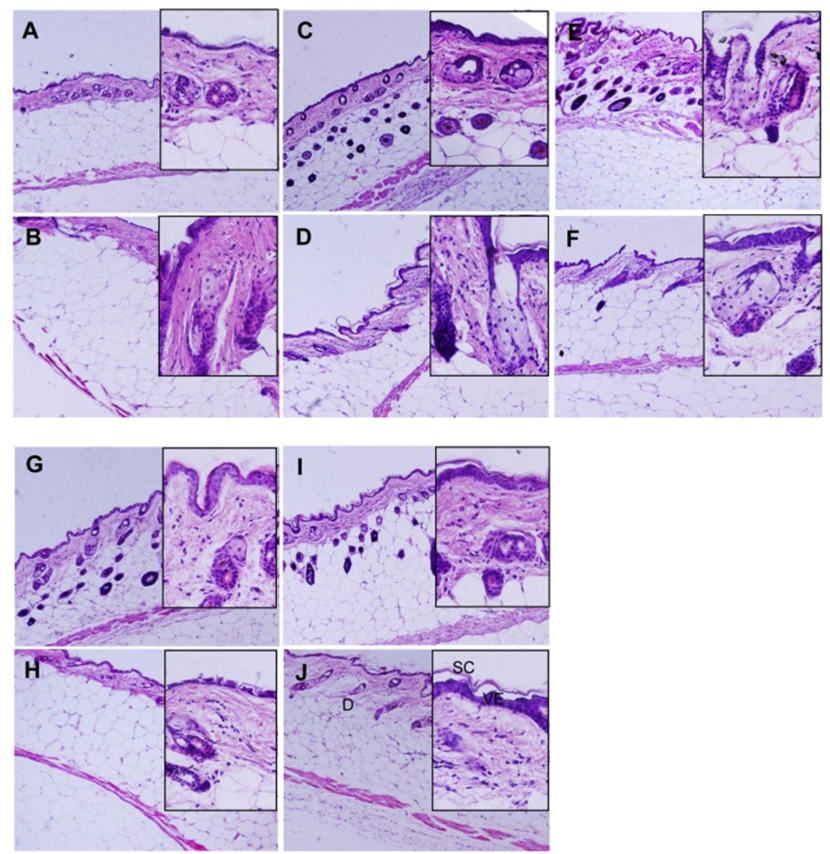

Fig. 4. Histological comparison of representative middorsal section of back skin which treated with DPHC. The integument of lean type mice (A, C, E, $\mathrm{G}, \mathrm{I})$ and obese type mice. (B, D, F, H, J) were represented. A and B, 0\% DPHC; C and D, 0.01\% DPHC; E and F, 0.1\% DPHC; G and H, 1\% DPHC; $\mathrm{I}$ and $\mathrm{J}, 10 \%$ DPHC. $\mathrm{H}$ and $\mathrm{E}$ staining, magnification $\times 100$ (insertion, $\times 400$ ). Collagen stained pale pink and muscle deep pink. Abbreviation: SC, stratum corneum; VE, viable epidermis; D, dermis, $\mathrm{H}$ and $\mathrm{E}$, hematoxylin-eosin. epidermal keratinocytes without increase in cell layer. More than $60 \%$ of the epithelial cells of viable epithelium showed diffused nuclear staining patterns and more cuboidal than $0 \%$ DPHC. Basement membrane was not different between the groups. In the lean group, hair follicle, a construct of cutaneous adnexa, was mostly located in the dermal matrix in $0 \%$ DPHC but was located in dermal adipocyte in all DPHC-treated groups (Fig. 4). On the other hand, in obesity group, most of the hair follicles were localized in the dermal matrix whether it was treated with DPHC or not (Fig. 4).

\section{DISCUSSION}

Histology of malignant integument is well described by Mecklenburg and his colleague (2013). Collagen crosslinks stabilize but become disorganized with aging (Duncan \& Leffell, 1997) and elastin is declined with age (Boss \& Seegmiller, 1981). Based on their criteria, histological characters of epidermis in all DPHC treated group was normal. In the histological analysis, DPHC did not cause pathological structural changes during obesity induction and obese conditions.

Hair coat is a character of mammalian skin although the density of it is less in humans. Mouse epidermis is approximately $10 \mu \mathrm{m}$ thick and has only $2-4$ cells layers thick. The stratum spinosum and stratum granulosum are not usually visible in mice (Mecklenbur et al., 2013). Proliferation of keratinocytes is restricted to epidermal progenitor cells (EPC). Self-generation and differentiation of EPC is strictly regulated by the signals combined external and internal information. Interestingly, the epithelial cells which has diffused nuclear staining patterns were increased both in the lean and obese mice after DPHC treatment. Besides, the epithelial cells were more cuboidal than 0\% DPHC. Some of the suggested roles of DPHC are to become a protector against radiation-induced cell damage and an anti-inflammatory agent (Ahn et al., 2011; Kang, 2014). Previously the extract of Ishige okamurae main compound 
of increased the viability of 3T3-L1 (Cha \& Cheon, 2013). Obesity induction caused increase in the number of fibroblast. However, DPHC groups, both in lean and obese groups, the number of fibroblast were decreased and showed statistical significance, and became similar between lean and obesity mice with $10 \%$ DPHC treatment. However, the intensities of collagen fibers and elastin fibers were increased by the DPHC. Previous studies showed that DPHC improved viability but suppressed abiogenesis of 3T3-L1 cell line and the extracts of Ishige okamurae increased the viability of 3T3-L1 (Cha \& Cheon, 2013). It means that DPHC, the main compound of Ishige okamura, may stimulate the activity of epithelial cells of mouse integument.

The ECM is an important component of niche and regulates stem cell behaviors (Scadden, 2006). ECM provides structural stability to the tissue and regulates intracellular signaling pathways that are important for cell fate decision. Dermal thickness decreases with age (Waller \& Maibach, 2005) and is accompanied by a decrease in both vascularity and cellularity (Suter-Widmer \& Elsner, 1996; Duncan \& Leffell, 1997). The amount of hyaluronic acid, interfibrillary ground substance, and elastin is declined with age (Boss \& Seegmiller, 1981; Castelo-Branco et al., 1998; Südel et al., 2005). Dermal fibroblasts, the main origin of ECM, use $\alpha 5 \beta 1$ or $\alpha v$ integrins to bind fibronectin, whereas interactions with collagens are mediated mainly by $\alpha 1 \beta 1, \alpha 2 \beta 1$, and $\alpha 11 \beta 1$ integrins (Eckes et al., 2010). Our results showed that the number of fibroblasts in a given field was decreased by DPHC both in lean and obese mice. However, interestingly, the intensity increased by DPHC during obesity induction. The intensity of elastic fibers was increased from lower concentration $(0.01 \%)$ in lean mice. The increase pattern of elastic fiber was different from the lean. It decreased at $0.01 \%$ DPHC, and increased from $0.1 \%$ DPHC and had statistical significance form 1\% DPHC. On the other hand, intensity of collagen fibers of obese mice was lower than lean mice in $0 \%$ group. However, the intensities of it became similar between lean and obesity mice by the treatment of DPHC.

Tight regulation of stem cell quiescence and activation is required to maintain a stable pool of stem cells enabling maintain through the lifetime (Blanpain \& Fuchs, 2009). Stem/progenitor cell niche is constructed with collagen rich ECM, veins, and nerves. The niche provides the localized environmental signals required to both maintain and mobilize stem/progenitor cells (Liu \& Engelhardtl., 2008; Yan \& Owens, 2008; Majesky et al., 2012). So far it is not clear whether DPHC stimulates the niche of progenitor cells of integument. But based on our results, it is suggested that DPHC may activate the integument fibroblast for ECM synthesis during obesity induction or in obese conditions.

It is a complex problem to understand the effects of functional molecules in integument during obesity induction and obese maintaining, because it is involved the microcirculatory system and lymphatics, the extracellular matrix and the presence of excess subcutaneous fat that bulges into the dermis. In here we did not show the regulation mechanisms of how DPHC is connected with the regulation of these compounds. However, it is important to understand the development of integument, or pathophysiology in a large variety of integument disorders. In this study, based on the results, it is suggested that a functional molecule such as DPHC can stimulate the activity of epithelial cells on cuboidal epithelium and the dermal blastocysts of mouse integument in both lean and obese condition to keep the stability of integument. DPHC also suppressed the increase the number of dermal blastocyst by obesity induction.

\section{ACKNOWLEDGEMENT}

We thanks to the help of Na Eun Kim, Hye-rin Kim, and Seung A Choi. This work was supported by the Sungshin University Un-Jeong Global Project research Grant of 2014. 


\section{REFERENCES}

Ahn M, Moon C, Yang W, Ko EJ, Hyun JW, Joo HG, Jee Y, Lee NH, Park JW, Ko RK, Kim GO, Shin T (2011) Diphlorethohydroxycarmalol, isolated from the brown algae Ishige okamurae, protects against radiation-induced cell damage in mice. Food Chem Toxicol 49:864-870.

Azzi L, El-Alfy M, Martel C, Labrie F (2005) Gender differences in mouse skin morphology and specific effects of sex steroids and dehydroepiandrosterone. J Invest Dermatol 124:22-27.

Blanpain C, Fuchs E (2009) Epidermal homeostasis: a balancing act of stem cells in the skin. Nat Rev Mol Cell Biol 10:207-217.

Boss GR, Seegmiller JE (1981) Age-related physiological changes and their clinical significance. West $\mathrm{J}$ med $135: 434$.

Cassisa A (2013) Pathophysiology of subcutaneous fat. G Ital Dematol Venereol 148:315-323.

Castelo-Branco C, Figueras F, Martinez de Osaba MJ, Vanrel JA (1998) Facial wrinkling in postmenopausal women. Effects of smoking status and hormone replacement therapy. Maturitas 29:75-86.

Cha S, Cheon YP (2013) Suppressive effects of an Ishige okamurae extract on 3T3-L1 proadipocyte differentiation. Dev Reprod 17:451-459.

Dubuc PU (1976) The development of obesity, hyperinsulinemia, and hyperglycemia in ob/ob mice. Metabolism 25:1567-1574.

Duncan KO, Leffell DJ (1997) Preoperative assessment of the elderly patient. Dermatol Clin 15:583-593.

Eckes B, Krieg T, Wickström SA (2014) Role of integrin signaling through integrin-linked kinase skin physiology and pathology. Exp Dematol 23:453-456.

Eckes B, Nischt R, Krieg T (2010) Cell-matrix interactions in dermal repair and scarring. Fibrogenesis Tissue Repair $3: 4$.
Heo SJ, Hwang JY, Choi JI, Han JS, Kim HJ, Jeon YJ (2009) Diphlorethohydroxycarmalol isolated from Ishige okamurae, a brown algae, a potent alpha-glucosidase and alpha-amylase inhibitor, alleviates postprandial hyperglycemia in diabetic mice. Eur J Pharmacol 615:252256.

Jeon Y, Song S, Kim H, Cheon YP (2013) Diphlorethohydroxycarmalol of Ishige okamurae and caffeine modified the expression of etracellular fibrillars during adipogenesis of mouse subcutaneous adipose derived stem cell. Devel Rperod 17:275-287.

Kang NJ (2014) Inhibitory effect and action mechanism of diphlorethohydroxycarmolol (DPHC) on the production of interleukin-6 in lipopolysaccharide (LPS)-stimulated murine macrophage RAW 264.7 cells. MS Thesis. Jeju University, Korea.

Liu X, Engelhardt JF (2008) The glandular stem/progenitor cell niche in airway development and repair. Proc Am Thorac Soc 5:682-688.

Majesky MW, Dong XR, Hoglund V, Daum G, Mahoney WM (2012) The adventitia: a progenitor cell niche for the vessel wall. Cells Tissues Organs 195:73-81.

Martini F (2004) Fundamentals of Anatomy and Physiology. Bejamin-Cummings, San Francisco,

McCallion R, Li WPA (1993) Dry and photo-aged skin: manifestations and management. J Clin Pharm Ther $18: 15$.

Mecklenburg L, Kusewitt D, Kolly C, Treuman S, Adams ET, Diegel K, Yamate J, Kaufmann W, Müller S, Danienko D, Bradley A (2013) Proliferative an nonproliferative lesions of the rat and mouse integument. $\mathrm{J}$ Toxicol Pathol 26:27S-57S.

Meyer W (2009) Hair follicles in domesticated mammals with comparison to laboratory animals and humans. In: Mecklenburg L, LineK M, tobin DJ (ed), Hair Loss Disorders in Domestic Animals. Wiley-Blackwell, Ames, IA, pp 43-64. 
Murillas R, Larcher F, Conti CJ, Santos M, Ullrich A, Jorcano JL (1995) Expression of a dominant negative mutant of epidermal growth factor receptor in the epidermis of transgenic mice elicits striking alterations in hair follicle development and skin structure. EMBO J 14:5216-5223.

Scadden DT (2006) The stem-cell niche as an entity of action. Nature 441:1075-1079.

Stern JL, Hagerman AE, Steinberg PD, Mason PK (1996) Phlorotannins-protein interactions. J Chem Ecol 22: 1877-1899.

Südel KM, Venzke K, Mielke H, Breitenbach U, Mundt C, Jasper S, Koop U, Sauermann K, Knussman-Harting E, Moll I, Gercken G, Young AR, Stäb F, Wenck H, Gallinat S (2005) Novel aspects of intrinsic and extrinsic aging of human skin: beneficial effects of soy extract. Photochem Photbiol 81:581-587.

Suter-Widmer J, Elsner P (1996) Age and irritation. In: Agner T, Maibah H (ed), The Irritant Contact Dermatitis Syndrome, CRC Press, Baca Raton, FL: pp 257-265.

Taylor DK, Bubier JA, Silva KA, Sundberg JP (2012) Development, structure, and keratin expression in C57BL/ 6 mouse eccrine glands. Vet Pathol 49:146-154.

Waller JM, Maibach HI (2005) Age and skin structure an function, a quantitative approach (I): blood flow, $\mathrm{pH}$, thickness, and ultrasound echogenicity. Skin Res Technol $11: 221-235$.

Yan X, Owens DM (2008). The skin: a home to multiple classes of epithelial progenitor cells. Stem Cell Rev 4:113-118. 